Note: This manuscript has been accepted for publication in Learning and Motivation. The published version can be found here: https://doi.org/10.1016/j.lmot.2020.101689

\title{
Differential and Experimental Approaches to Studying Intelligence in Humans and Non-Human Animals
}

\author{
Alexander P. Burgoyne, Jason S. Tsukahara, Christopher Draheim, \& Randall W. Engle \\ Georgia Institute of Technology
}

Funding. This work was supported by Office of Naval Research Grants N00173-20-2-C003 and N00173-20-P-0135 to Randall W. Engle. 


\begin{abstract}
Why do some individuals learn more quickly than others, or perform better in complex cognitive tasks? In this article, we describe how differential and experimental research methods can be used to study intelligence in humans and non-human animals. More than one hundred years ago, Spearman (1904) discovered a general factor underpinning performance across cognitive domains in humans. Shortly thereafter, Thorndike (1935) discovered positive correlations between cognitive performance measures in the albino rat. Today, research continues to shed light on the underpinnings of the positive manifold observed among ability measures. In this review, we focus on the relationship between cognitive performance and attention control: the domain-general ability to maintain focus on task-relevant information while preventing attentional capture by task-irrelevant thoughts and events. Recent work from our laboratory has revealed that individual differences in attention control can largely explain the positive associations between broad cognitive abilities such as working memory capacity and fluid intelligence. In research on mice, attention control has been closely linked to a general ability factor reflecting route learning and problem solving. Taken together, both lines of research suggest that individual differences in attention control underpin performance in a variety of complex cognitive tasks, helping to explain why measures of cognitive ability correlate positively. Efforts to find confirmatory and disconfirmatory evidence across species stands to improve not only our understanding of attention control, but cognition in general.

Keywords: Individual differences, differential psychology, intelligence, attention control, executive attention
\end{abstract}




\section{Differential and Experimental Approaches to Studying Intelligence \\ in Humans and Non-Human Animals}

The scientific study of cognition and behavior involves a range of methods and disciplines, from the experimental tradition of manipulating variables to the differential tradition of studying individual differences. While psychological research on humans has seen the development of sophisticated experimental and differential methods, non-human animal research has largely neglected differential methods in favor of the experimental approach. In this article, we discuss research on individual differences in intelligence in humans and non-human animals to bridge the gap between these two disciplines.

Ideally, our research questions and hypotheses should drive our methods of inquiry. It is all too common, however, for the methods with which we are most familiar to drive the types of questions we ask, and in turn, the hypotheses we form. The experimental approach is often used to identify causal factors influencing cognition and behavior at the group level, addressing questions such as, "how does manipulating variable X affect behavior Y?" The behavior in question is typically performance on a single task that, putatively, reflects a psychological construct. The differential approach, on the other hand, is used to answer questions about how individuals differ in terms of cognitive ability, personality, motivation, interests, and so on, and how these factors relate to each other and predict performance in a domain. In differential studies, psychological constructs are typically reflected by multiple tasks, giving rise to latent variables that are often regarded as more process pure than single tasks (Ambrosini et al., 2019). Whereas variability across individuals is the phenomenon of interest for differential researchers, the question of how individuals differ is often ignored by experimental researchers. Because experimentalists who study non-human animals may be less well acquainted with the merits of 
individual differences research, in this article, we show how the differential approach can be used to study intelligence across species, and how combining the methods of the "experimentalist" and the "correlator" (Cronbach, 1957) can advance our understanding of cognition and behavior.

\section{Intelligence Differences in Humans}

Why do some individuals learn more quickly than others, or perform better in complex cognitive tasks? These questions have been at the center of differential psychology since its inception. Over a century ago, Charles Spearman (1904) discovered that students who performed well in one course often performed well in other courses, too. Spearman (1904) computed the correlations between students' grades in Classics, Math, English, French, and Music, and found that they were uniformly positive and relatively high. To explain why some students performed well across the board while others performed poorly, Spearman (1904) introduced the field to factor analysis, a statistical technique for simplifying patterns of covariance among observed measures by describing them in terms of a smaller number of unobserved or latent variables (Gottfredson, 1998). Factor analysis revealed a domain-general ability factor that explained the positive relationships between course grades. That is, scores on the general factor accounted for substantial variance in course performance, ranging from $49 \%$ in Music to $98 \%$ in Classics. This led Spearman to conclude that domain-general ability contributed to individual differences across cognitive domains, while acknowledging the role of domain-specific factors. As Spearman (1904) stated: "all branches of intellectual activity have in common one fundamental function (or group of functions), whereas the remaining or specific elements of the activity seem in every case to be wholly different from that in the others" (p. 294). 
Today, research has convincingly established that scores on cognitive ability tests correlate positively, implying the existence of a domain-general ability, termed the " $g$ factor" (Carroll, 1993; Jensen, 1998; McGrew, 2009). The $g$ factor, often interpreted as representing general intelligence, is a higher-order statistical factor that accounts for the relationships among broad cognitive abilities, such as fluid intelligence, crystallized intelligence, and working memory capacity. Although the $g$ factor is ubiquitous in intelligence research, cognitive scientists continue to search for the 'common function' (or group of functions) responsible for the positive manifold - the positive relationships observed among cognitive ability measures (see, e.g., Kovacs and Conway's [2016] process overlap theory; Dickens and Flynn's [2001] gene-environment multiplier theory; and Savi, Marsman, van der Maas, and Maris's [2019] theory of evolving networks).

Our research investigates the underlying mechanisms of $g$. More specifically, one of our goals is to find sources of variance across individuals that are common to a variety of complex cognitive tasks, helping to explain why performance measures correlate positively. To do so, we test large samples of human participants (e.g., 300-500) representing a wide range of ability using a variety of measures thought to reflect cognitive constructs. We then use factor analysis and structural equation modeling to elucidate relationships between latent variables representing broad cognitive abilities. We use latent variables (i.e., unobserved variables) because they capture variance common to a set of observed measures and are therefore less affected by measurement error and closer to the theoretical constructs of interest (Kline, 2015). To supplement this correlational approach, we also use experimental manipulations to determine the conditions under which task performance taps particular cognitive constructs, such as attention control. Broadly speaking, our research combines the differential and experimental traditions- 
two methods of inquiry that were historically siloed (Cronbach, 1957) — to shed light on the cognitive processes underlying complex task performance.

\section{The Executive Attention View}

Research from our laboratory has revealed that one of the reasons individual differences in broad cognitive abilities correlate positively is because complex cognitive tasks demand attention control (Burgoyne \& Engle, in press; Engle, 2018; Shipstead et al., 2016). Attention control refers to the domain-general ability to maintain focus on task-relevant information while preventing attentional capture by task-irrelevant thoughts and events. This latter function is associated with the terms inhibition, blocking, ignoring, and tagging for non-retrieval. Attention control guides thoughts and behaviors in a goal-driven manner, and is particularly important under conditions of distraction or interference. It is assumed that the control of attention requires effort and thus constitutes mental work.

The theoretical contribution of attention control to cognitive performance is closely tied to its role coordinating two functions: maintenance and disengagement (Shipstead et al., 2016). Maintenance refers to the set of cognitive operations responsible for maintaining access to goalrelevant information. Successful maintenance is threatened by distraction and interference due to internal thoughts (e.g., mind wandering) and external events (e.g., a nearby conversation). Disengagement, by contrast, refers to the cognitive operations responsible for removing nolonger relevant information from active processing. Disengagement is necessary when, for example, prior learning interferes with one's ability to perform adaptively under new circumstances. We view intentional maintenance and disengagement as two distinct mechanisms by which attention control is deployed. As we will show, maintenance and disengagement play complementary roles in most cognitive tasks. 
Consider the contribution of maintenance and disengagement to performance in complex span tasks, which are often used to measure working memory capacity. Complex span tasks challenge participants to keep track of memoranda while completing a secondary processing task. For example, in Operation Span (Unsworth et al., 2005), the participant is shown letters they must memorize in alternation with simple math equations they must solve (Figure 1a). After a series of letters and equations, the participant must recall the letters in the order they were presented. Information maintenance is critical to remember the list of letters, especially because the secondary processing task creates interference and prevents effective rehearsal of the memoranda (Kane et al., 2007). That said, disengagement from no-longer-relevant information also plays a role, because the participant must be careful not to recall letters from previous trials.

a)

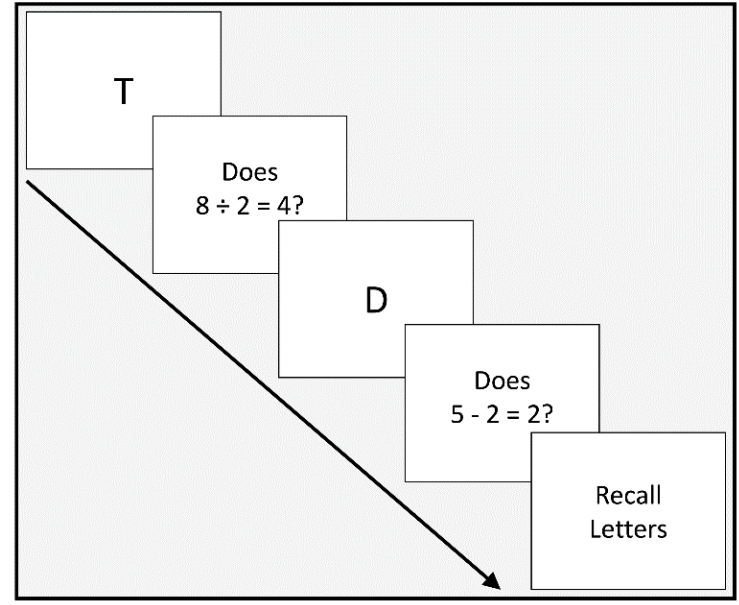

b)

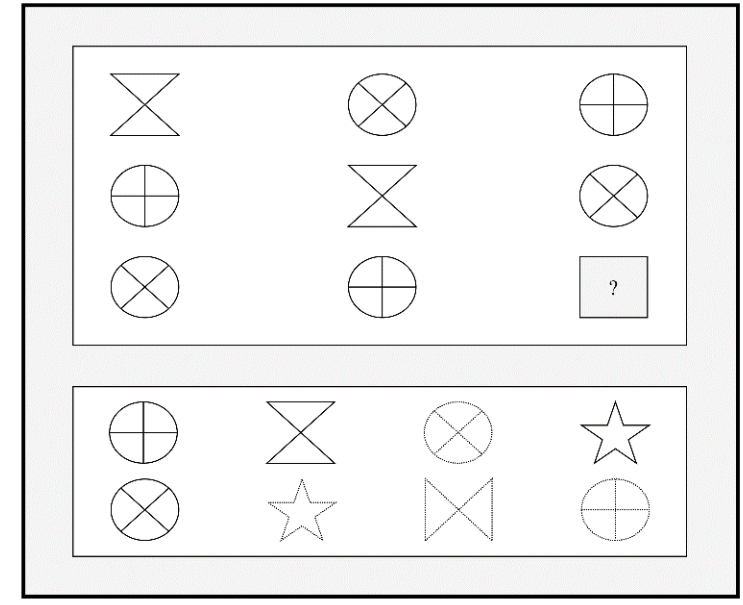

Figure 1. a) The Operation Span task (Unsworth et al., 2005) is a complex span task used to measure working memory capacity. The participant is shown a letter to be remembered, followed by a math equation to be solved. After a series of letters and equations, the participant must recall the letters in the order they were presented. b) An example item similar to those used in Raven's Matrices (Raven \& Court, 1998), a test of fluid intelligence. The participant is shown a $3 \times 3$ grid of symbols following a pattern, but the symbol in the bottom right corner is missing. The participant must select from a set of options the symbol that best completes the pattern. 
Whereas working memory tests emphasize maintenance over disengagement, fluid intelligence tests depend on the ability to disengage from no-longer-relevant information during problem solving. For instance, in Raven's Matrices (Raven \& Court, 1998), the participant is shown a $3 \times 3$ grid of symbols that follow a pattern, but the symbol in the bottom-right corner is missing (Figure 1b). To solve the problem, the participant must develop and test hypotheses about the pattern characterizing the grid of symbols and select the response option that best completes the pattern. Disengagement from hypotheses that have already been ruled out is important, otherwise the participant is left perseverating on incorrect solutions at the expense of testing time. Of course, disengagement is not the only mechanism that gives rise to individual differences in fluid intelligence test performance. We would argue, however, that disengagement is the key factor that links fluid intelligence with attention control.

According to the executive attention theory of working memory capacity (Engle, 2002; Engle \& Kane, 2004), variation in working memory capacity largely reflects individual differences in the ability to control attention to do the mental work to maintain task-relevant information and disengage from no-longer relevant information. The working memory system is classically defined as the interplay between a central executive (i.e., controlled-attention) component and short-term storage components, including the visuospatial sketchpad, which is responsible for visual information, and the phonological loop, which is responsible for speechbased information (Baddeley \& Hitch, 1974; Baddeley, 1992). Subsequent models of working memory included an episodic buffer, which is controlled by the central executive and is responsible for the integration of information (Baddeley, 2000). It is our argument that the attentional component of the working memory system primarily accounts for working memory capacity's predictive power. 
Evidence for the executive attention view is provided by research showing that the central executive component of working memory drives its relationship with broad cognitive abilities such as fluid intelligence. For example, Engle et al. (1999) assessed relationships between latent variables representing working memory capacity, short-term memory, and fluid intelligence in a sample of 133 participants (Figure 2). Working memory capacity and short-term memory were strongly correlated, $r=.68$, indicating that they shared approximately $46 \%$ of their reliable variance. This shared variance primarily reflects memory storage demands common to short-term memory and working memory tasks. The correlation fell short of unity, indicating that there was unique variance to distinguish working memory capacity from short-term memory. The unique variance specific to working memory capacity likely represents the additional burden placed on attention control when performing complex span tasks, because dual-tasks demand greater information maintenance in the context of interference than simple short-term storage tasks do. Critically, the unique contribution of working memory capacity to fluid intelligence was large and significant, whereas the unique contribution of short-term memory to fluid intelligence was near zero and non-significant. This suggests that individual differences in attention control, and not short-term storage, drive the correlation between working memory capacity and fluid intelligence. Several other large-scale latent variable analyses have corroborated this finding (see, e.g., Conway, et al., 2002; Kane et al., 2004). 


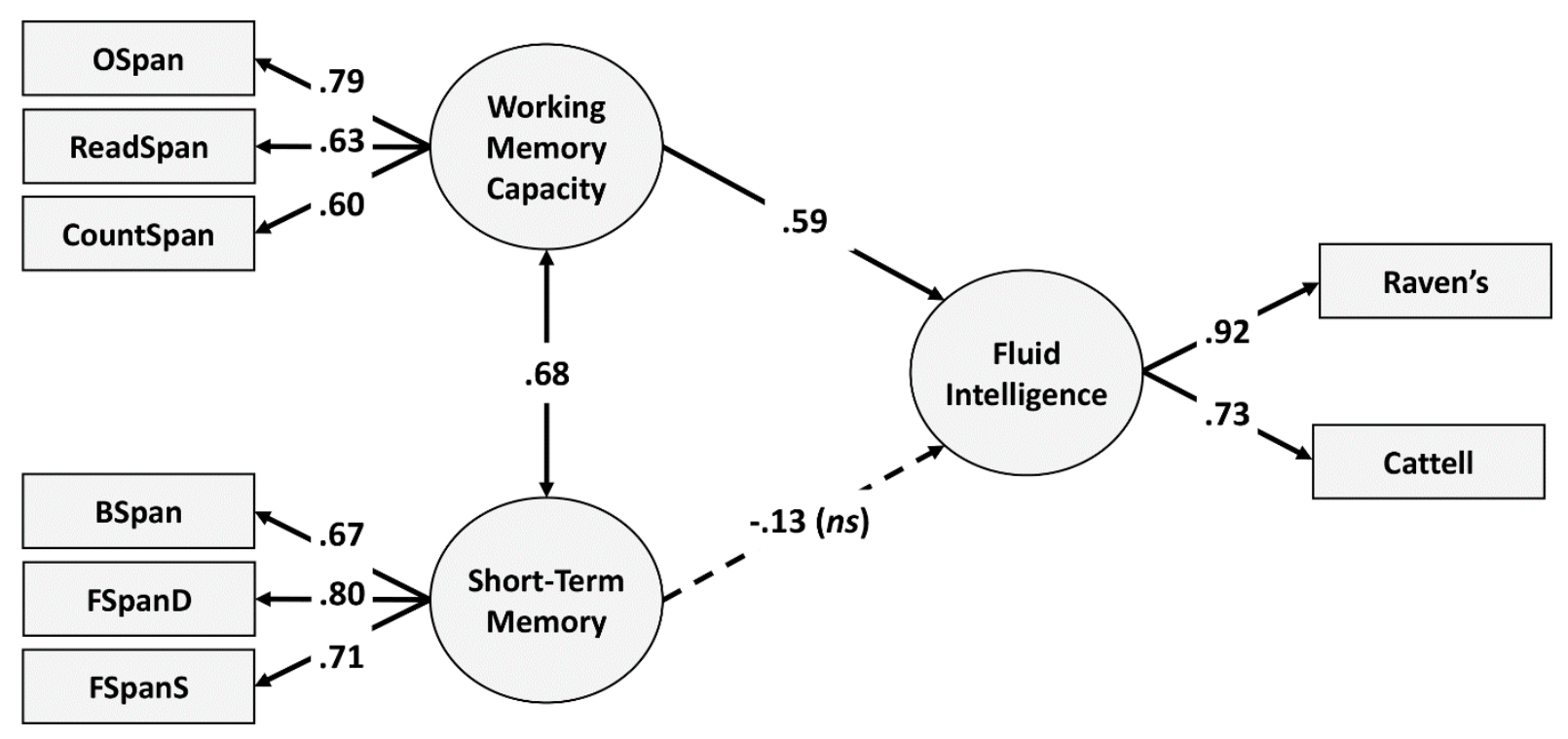

Figure 2. In this structural equation model, latent factors are depicted in ovals and observed measures are depicted in rectangles. Working memory capacity and short-term memory were strongly correlated (.68), as indicated by the value along the double-headed arrow. The contribution of working memory capacity to fluid intelligence (.59) was substantial and significant after accounting for short-term memory. By contrast, the contribution of short-term memory to fluid intelligence $(-.13, n s)$ was non-significant after accounting for working memory capacity. This pattern of results suggests that controlled attention drives the relationship between working memory capacity and fluid intelligence. Adapted from Engle et al. (1999).

Evidence for the executive attention view is also provided by studies of performance on tasks requiring controlled processing, particularly under conditions in which interference leads to response tendencies that are in conflict with task goals (Engle, 2002). The rationale is that if individual differences in working memory capacity primarily reflect controlled attention, then the effects of working memory capacity on task performance should still be observed in contexts that demand controlled processing but make minimal demands on memory storage and retrieval.

For example, in a dichotic listening task (Figure 3a), participants are presented two auditory streams of words simultaneously, one to each ear. Their task is to shadow (i.e., say aloud) the words presented to one ear while ignoring the words presented to the other. At some 
point during the task, the participant's name is unexpectedly presented to the ignored ear. Typically, around one-third of people report hearing their name- an effect referred to as the cocktail party phenomenon (Moray, 1959). Conway, Cowan, and Bunting (2001) found that participants with low working memory capacity were more likely to report hearing their name in the ignored ear than participants with high working memory capacity. This suggests that high working memory capacity individuals are better able to control attention to block or inhibit distractions than low working memory capacity individuals.

Another task that makes use of a salient distractor is the antisaccade task (Hallet, 1978; Figure $3 \mathrm{~b}$ ). In the antisaccade task, a flashing cue is presented on one side of the computer screen, and the participant must rapidly make a saccadic eye movement to the opposite side of the screen to detect a briefly presented letter. To successfully perform the task, the participant must inhibit a reflexive response - don't look at the flashing cue — and generate a volitional eye movement in the opposite direction. By contrast, in the prosaccade version of the task, the participant simply looks toward the flashing cue - there is no need to inhibit a reflexive response. Kane, Bleckley, Conway, and Engle (2001) tested 203 high and low working memory capacity participants on the antisaccade and prosaccade tasks. Overall, they found no differences between high and low ability participants in the prosaccade task. In the antisaccade task, however, high working memory capacity participants made fewer saccade errors (i.e., looking toward the flashing cue), and when they did make saccade errors, they recovered faster from them. 
a)

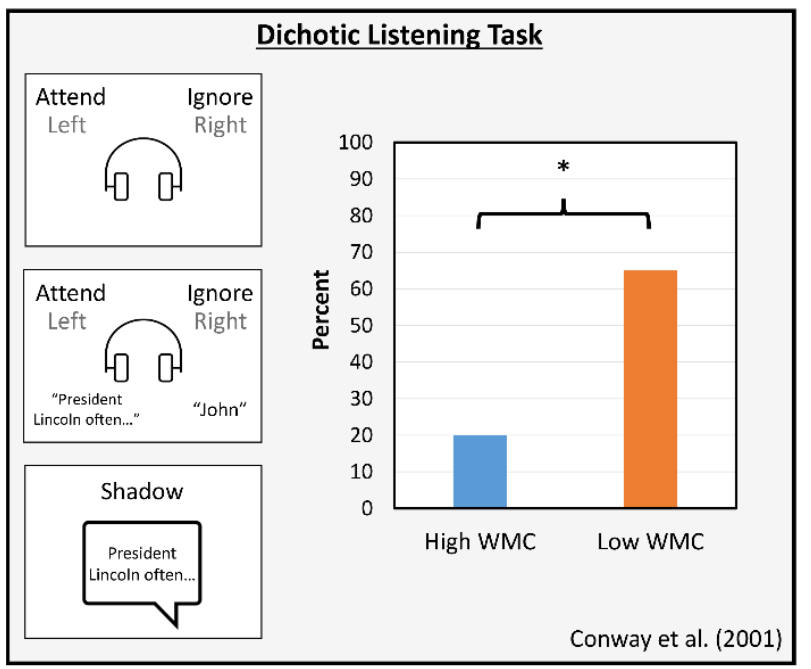

b)

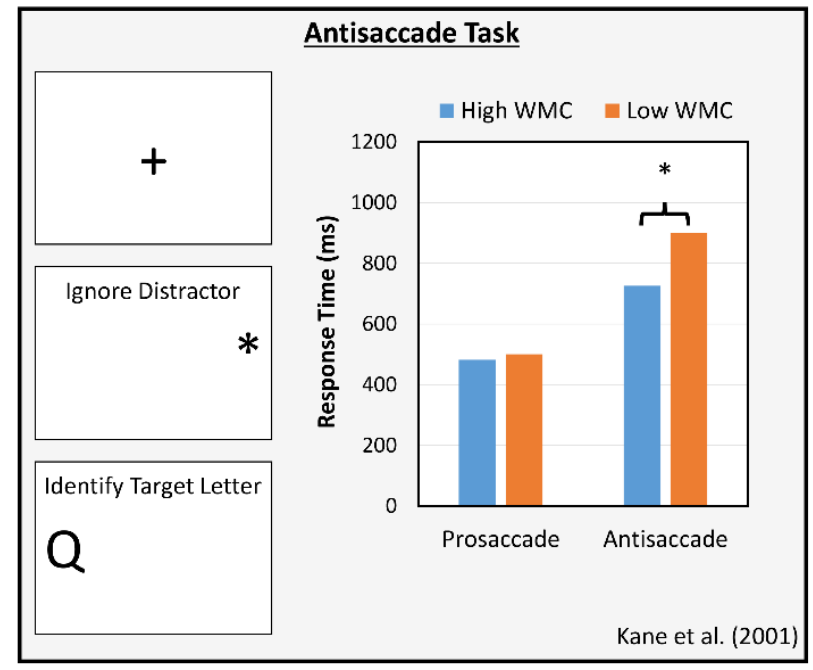

Figure 3. a) Example of the dichotic listening task and results from Conway et al. (2001). Low working memory capacity (WMC) participants were more likely to detect the presentation of their name in the unattended ear than high working memory capacity participants. b) Example of the antisaccade task and results from Kane et al. (2001). Low working memory capacity participants were significantly slower to perform the antisaccade task than high working memory capacity participants. The groups did not differ in prosaccade task performance.

One last example of a task that requires controlled processing is the flanker task (Eriksen \& Eriksen, 1974). In the flanker task, participants are presented with a string of letters and must indicate the central letter while ignoring the flanking letters. On congruent trials (e.g., $H H H H H$ ), the central letter is the same as the flanking letters, whereas on incongruent trials (e.g., $H H S H H$ ), the central letter differs. Heitz and Engle (2007) hypothesized that attention functions like a dynamic spotlight during performance: initially, visual attention is diffuse and captures the whole display, but as the trial progresses, attention becomes progressively more focused on the central letter to the exclusion of the flankers. Additionally, they hypothesized that individuals with greater attention control would be quicker to constrain visual attention. To test this hypothesis, they had high and low working memory capacity participants complete flanker trials with different response deadlines. Heitz and Engle (2007) found that both groups reached 
asymptotic performance when given plenty of time to respond, but that when response times were constrained, high working memory capacity participants outperformed low working memory capacity participants (Figure 4). This suggests that participants with greater attention control were quicker to constrain their attention to focus on task-relevant information and ignore distractors.

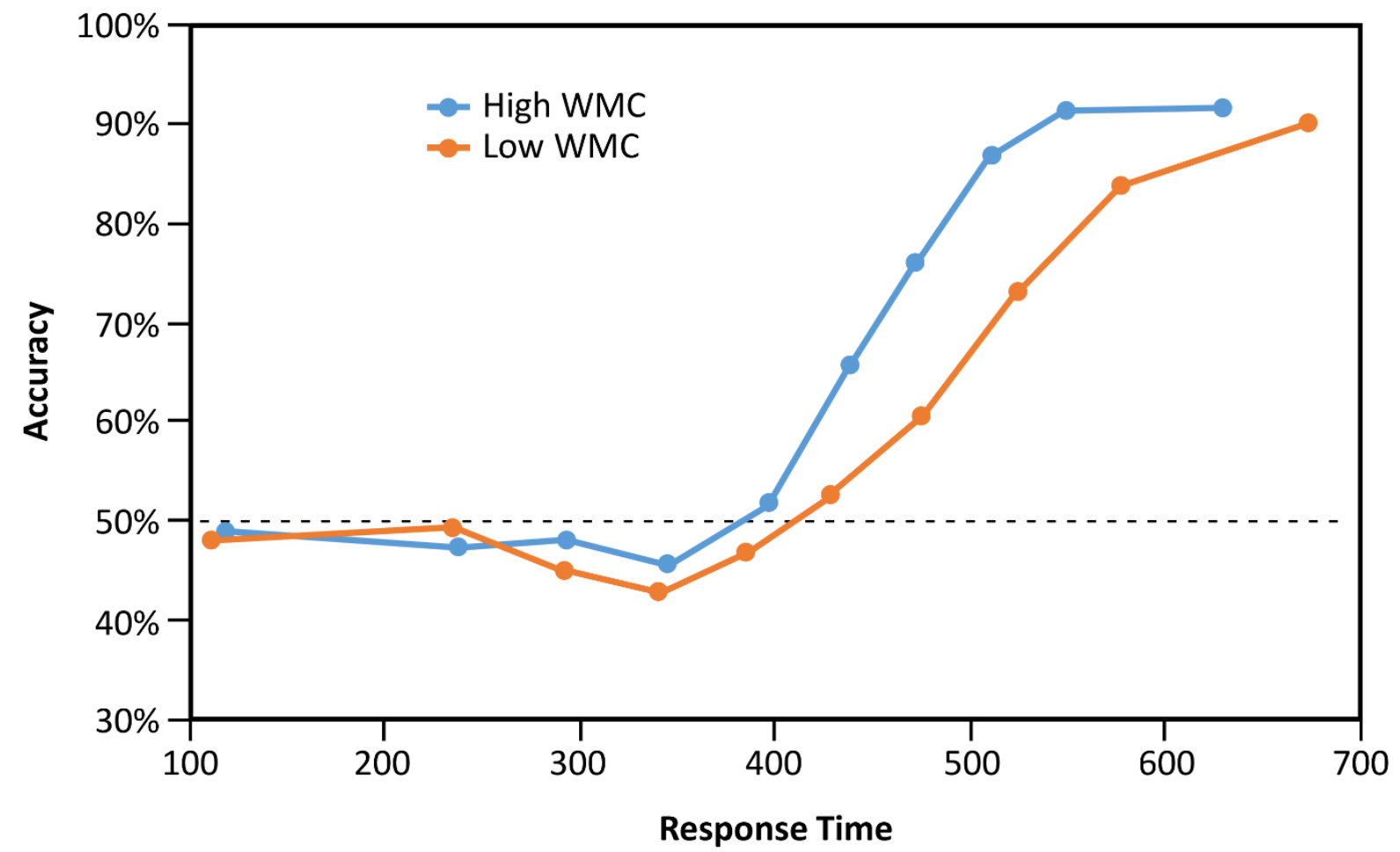

Figure 4. Conditional accuracy functions for incongruent trials from the flanker task. As the response time deadline increased, high working memory capacity (WMC) participants reached peak performance before low working memory capacity participants. Adapted from Experiment 3 of Heitz and Engle (2007).

Perhaps the most compelling recent evidence for the executive attention view is provided by Draheim et al. (2020), who found that individual differences in attention control could explain the relationship between working memory capacity and fluid intelligence in a sample of 396 
participants. Attention control was measured using a battery of accuracy-based tests that challenged participants to focus on task-relevant information in the context of interference and distraction, including versions of the antisaccade and flanker tasks described above. Draheim et al. (2020) found that latent factors representing working memory capacity and fluid intelligence were strongly correlated, but that attention control could fully mediate this relationship. That is, the relationship between working memory capacity and fluid intelligence was no longer statistically significant after accounting for attention control (Figure 5). Draheim et al.'s (2020) approach represents a more rigorous test of Engle et al.'s (1999) findings, with both studies converging on the same conclusion: the primary driver of the relationship between working memory capacity and fluid intelligence is attention control.
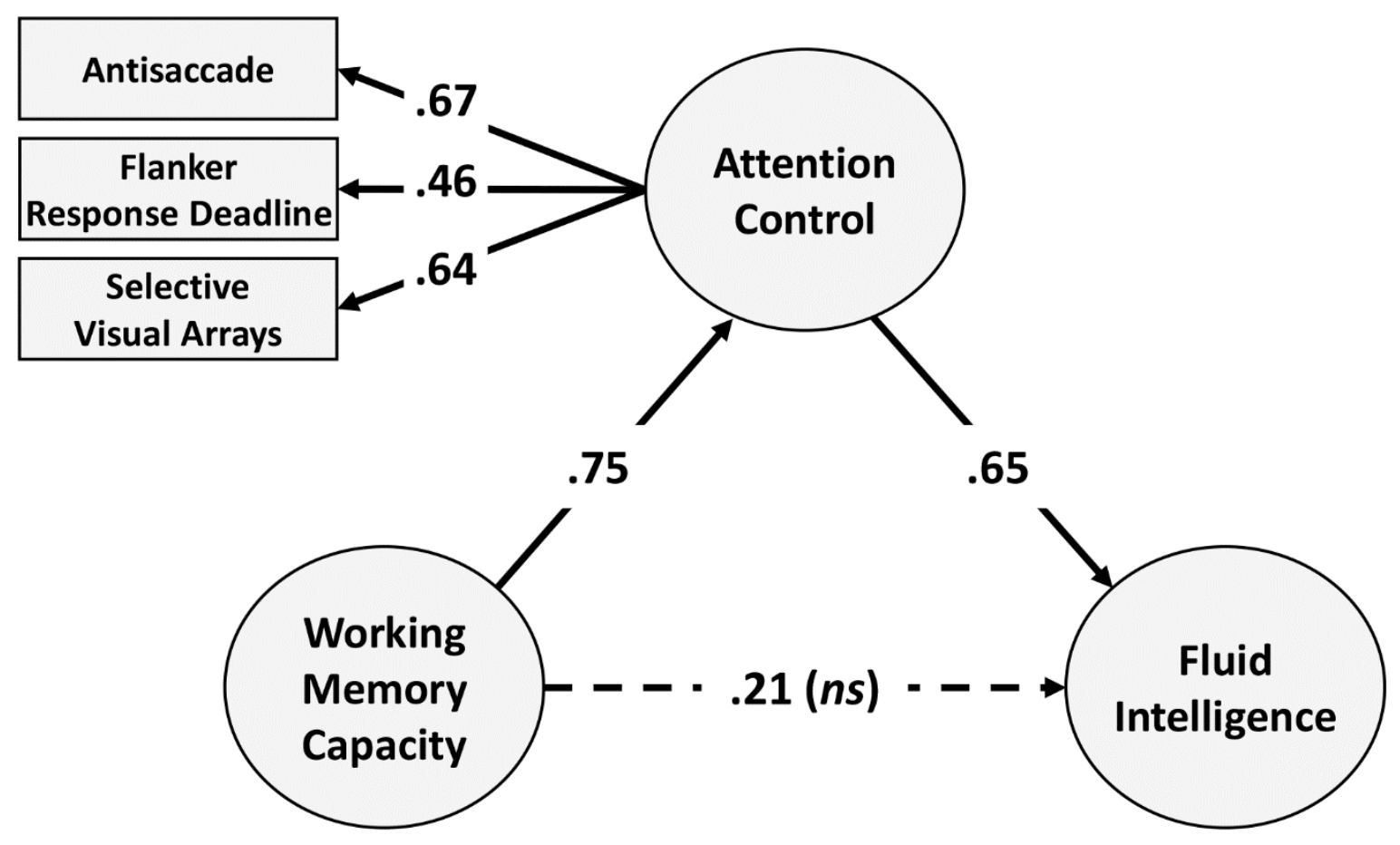

Figure 5. This model depicts attention control fully mediating the relationship between working memory capacity and fluid intelligence. As can be seen, working memory capacity was strongly related to attention control (.75), and attention control was strongly related to fluid intelligence (.65). Although there is usually a strong relationship between working memory capacity and fluid intelligence, this model shows that working memory capacity does not predict fluid intelligence $(.21, n s)$ after accounting for attention control. The results of this model therefore 
indicate that attention control drives the relationship between working memory capacity and fluid intelligence. Adapted from Draheim et al. (2020).

Based on the executive attention view, we would expect that attention control not only explains the relationship between working memory capacity and fluid intelligence, but other cognitive functions, as well. For instance, one of the oldest findings from intelligence research is that general sensory discrimination ability correlates strongly with general intelligence (Spearman, 1904). Sensory discrimination refers to the ability to make fine perceptual judgments about auditory, visual, and tactile stimuli. For example, imagine you hear two tones and must decide which has a higher pitch, or see two lines of similar length and must determine which is longer. Consistent with Spearman's observation, Tsukahara et al. (2020) found that sensory discrimination ability was strongly related to working memory capacity and fluid intelligence at the latent level in two samples of 331 and 392 participants. Moreover, attention control largely mediated these positive relationships. That is, the relationships between both working memory capacity and fluid intelligence with sensory discrimination ability were non-significant after accounting for attention control. Tsukahara et al.’s (2020) work represents a major advance, because it suggests that attention control may explain the positive manifold not only among higher-order cognitive constructs such as working memory capacity and fluid intelligence, but also among more "primitive" or lower level constructs, such as general sensory discrimination ability as measured by a variety of auditory and visual tasks.

Taken together, research on humans points to attention control as an explanatory mechanism and statistical mediator of the relationships between working memory capacity, fluid intelligence, and sensory discrimination ability. That said, we view attention control as just one piece of the puzzle underlying individual differences in intelligence. In the next section, we 
review converging evidence from research on non-human animals linking attention control to performance in cognitive tasks.

\section{Intelligence Differences in Animals}

Thirty-one years after Spearman's (1904) discovery of a general ability factor in humans, Thorndike (1935) discovered positive correlations between cognitive performance measures in the albino rat. Since then, some researchers have continued to search for reliable individual differences in cognitive ability in non-human animals. The past twenty years have been particularly productive: it has now been established that a general factor underpins learning abilities in genetically heterogeneous mice across a variety of tasks such as maze navigation, sensory discrimination, and fear conditioning (Matzel et al., 2003; see also Galsworthy et al., 2002). This general factor, which is conceptually similar to general intelligence in humans, accounts for around one third of the variance in performance in tasks tapping different sensory, motor, motivational, and information processing demands (Matzel et al., 2003). Researchers have also discovered evidence for a general factor in border collies (Arden \& Adams, 2016) and wild Australian magpies (Ashton et al., 2018). Others have found that individual differences in problem solving can be reliably measured in spotted hyenas (Johnson-Ulrich, Holekamp, \& Hambrick, 2020) and great tits (Cole, Cram, \& Quinn, 2011). The evidence to date suggests that individual differences in intelligence in non-human animals can be measured both in the laboratory and in the wild, and that the positive manifold among ability measures is not a phenomenon that is unique to humans.

Just as human intelligence researchers seek to understand what the $g$ factor is, animal cognition researchers search for common sources of variance that contribute to performance across cognitive tasks. In this section, we focus on research led by Lou Matzel and colleagues, 
with an emphasis on evidence linking attention control to general intelligence in mice. Before reviewing the evidence, however, it is worth noting some of the differences between research on mice versus humans.

First, unlike in human studies, research on mice affords the experimenter control over environmental influences throughout the animals' lifespan. In the studies described below, laboratory mice are given the same amount of handling, food, and light exposure. This ensures that most of the variability across individuals is attributable to genetic influences, which is particularly useful for behavioral genetic research (see, e.g., Kolata et al., 2010). The mice in these studies are genetically heterogeneous, and although they are laboratory bred, they exhibit similar levels of genetic variation to wild mouse populations (Crawford et al., 2020; Aldinger et al., 2009). Genetic variation is important, because individual differences arise from the joint product of genes and the environment (Plomin, 2017)—in the absence of genetic differences, there would be little to no variation across individuals to observe.

Second, although the tasks that are used to measure cognitive functioning in mice are sometimes conceptually similar to those that are used for humans, there are also potentially important differences to consider. Many of the intelligence tests used for mice assess route learning ability or problem solving using maze tasks or puzzles. As one example, in the Lashley maze task (Figure 6a), the mouse must efficiently navigate a maze from a start box to a goal area containing a food reward (Crawford et al., 2020). After completing a few practice trials, the measure of performance is the number of navigational errors made en route to the food. On critical "reversal" trials (Figure 6b), however, the mouse is placed in the goal area instead of the start box. The food cup in the goal area is empty, and the mouse's task is to find their way back to the start box to retrieve a food reward. Two measures of performance are obtained from 
reversal trials: navigational errors made en route to the food, and the number of times the mouse approaches the empty food cup in the goal area, which is indicative of perseverating on a previously learned association. Whereas performance on non-reversal trials is used as a measure of route learning ability, performance on reversal trials is used as a measure of attention control, and in particular, disengagement from no-longer-relevant information.

a)

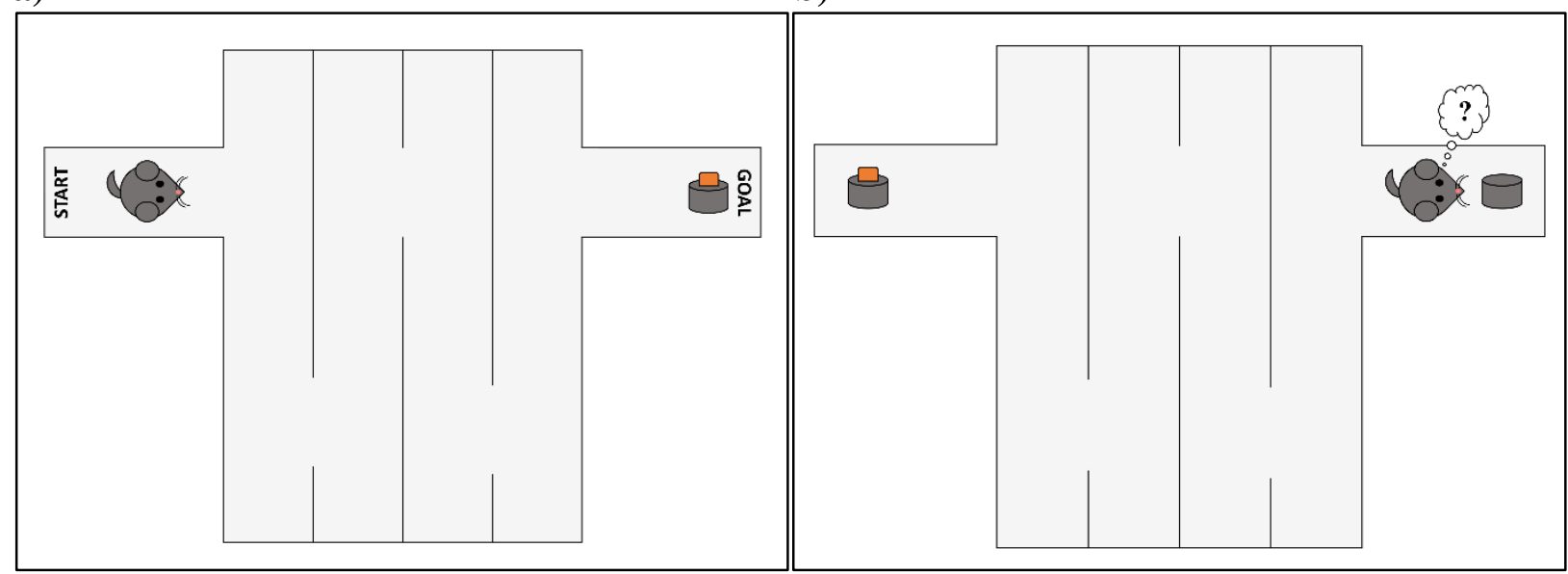

Figure 6. a) Standard administration of the Lashley maze task used by Crawford et al. (2020) to measure route learning ability. The mouse must navigate the maze from the start area to the goal area to obtain the food reward. (b) "Reversal" trials in the Lashley maze task used to measure disengagement from no-longer-relevant information. The mouse is placed in the goal area facing an empty food cup and must navigate the maze to the former start area to obtain the food reward.

In a recent series of studies, Crawford et al. (2020) found that attentional disengagement was strongly related to individual differences in general intelligence in mice. Crawford et al. (2020) obtained eight measures of cognitive ability from 74 mice: six were measures of learning ability, and two were measures of attentional disengagement taken from Lashley maze reversal trials. All measures correlated positively, suggesting a common source of variance across the tasks. Crawford et al. (2020) conducted a principal components analysis on the eight measures to 
examine their $g$ loadings, reflecting each measure's relationship to the general ability

underpinning performance on the task battery. Even though the battery was dominated by six measures of learning ability, the two measures of attention control had the highest loadings on the general factor. This suggests that there was a close connection between general intelligence and attention control, as measured by the ability to disengage from previously-relevant information. Consistent with this finding, Crawford et al. (2020) performed a quartile split on the sample, identifying smart and dull mice based on their performance on the six learning measures. This extreme-groups design revealed significant differences in performance on Lashley maze reversal trials (Figure 7), suggesting that mice with greater general ability were better able to control attention in service of task performance.

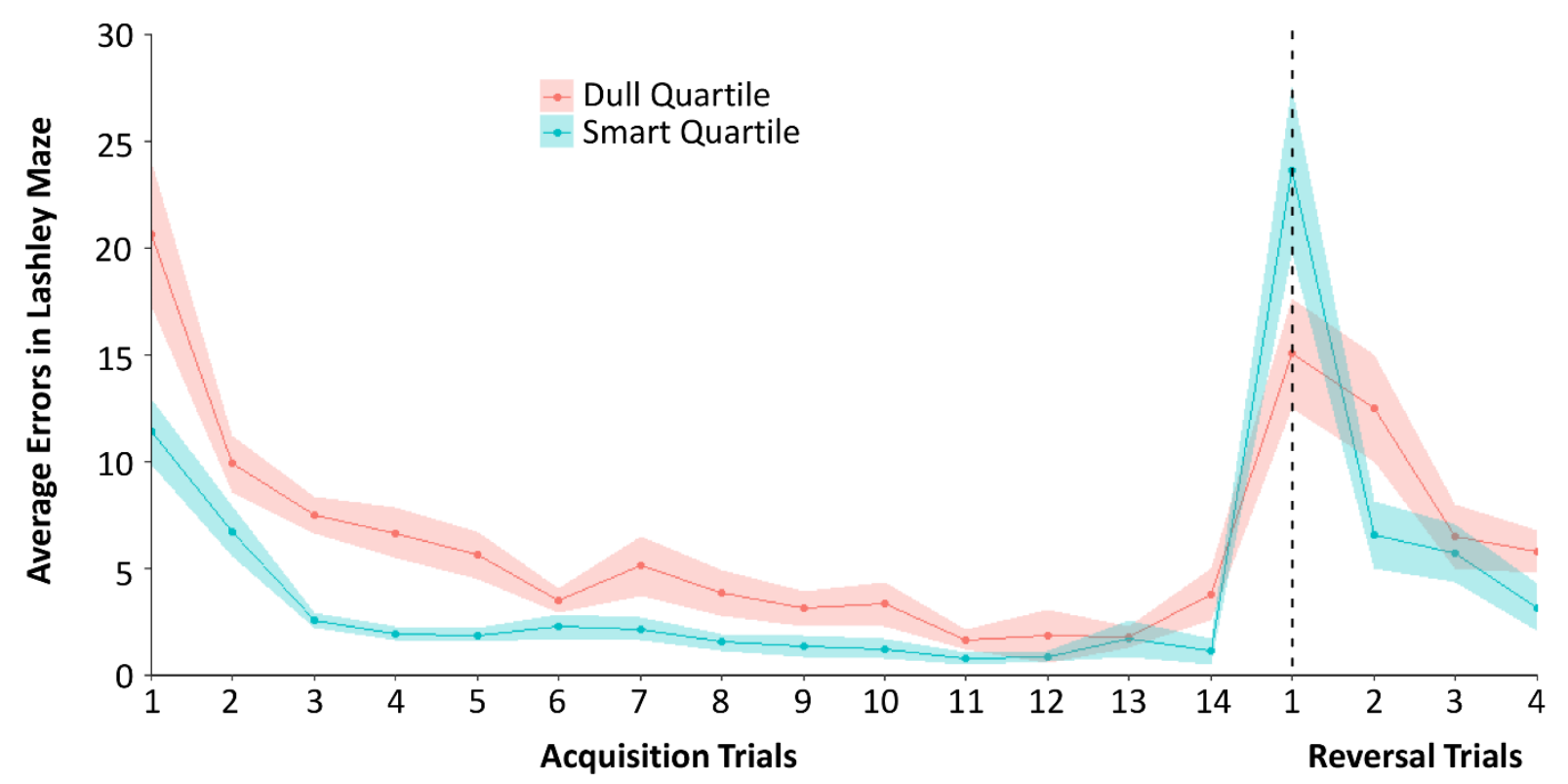

Figure 7. Performance on Lashley maze acquisition trials and reversal trials by smart and dull mice. During the acquisition phase, smart mice made fewer errors. During the reversal phase, smart mice initially made more errors than dull mice (see Reversal Trial 1). By the second reversal trial, however, smart mice had adapted to the new route better than dull mice. Error bars represent \pm 1 standard error. Adapted from Crawford et al. (2020). 
Whereas the Lashley maze reversal trials Crawford et al. (2020) used to measure attention control in mice tapped disengagement from no-longer-relevant information, a study by Kolata et al. (2005) revealed a similar pattern of results using a test that tapped information maintenance amidst interference. Similar to a complex span test administered to humans, the mice were challenged to navigate two radial-arm mazes in alternating order. After training on each maze individually, the mice had to collect food pellets from the first maze before being moved to the second. There, they collected more food before they were moved back to the first maze to resume their search. This alternating procedure continued until all food pellets had been collected. To successfully perform the task, the mice had to keep track of information from the first maze while completing the second, and vice versa. Kolata et al. (2005) found that performance on the dual-maze task was strongly related to overall performance on a battery of cognitive ability tests $(r=.60)$, establishing a link between general ability and the maintenance of information via attention control.

Consistent with the executive attention view of working memory capacity (Engle \& Kane, 2004), the relationship between dual-maze performance and general intelligence seems to be driven by the active maintenance of information in the context of interference, rather than passive short-term storage. Kolata et al. (2007) tested the relationship between short-term memory duration and general intelligence using a single maze and an unfilled delay between moves. Specifically, after the mice had made several navigational choices in the maze, they were placed in confinement for a variable duration before being returned to the maze to continue foraging. Although the error rate increased with the duration of confinement, performance in the short-term-storage maze task was unrelated to overall cognitive ability (Kolata et al., 2007). These results align with those of Engle et al. (1999) in humans - both indicate that the controlled 
processing component tapped by measures of working memory capacity (i.e., attention control) is more strongly related to higher-order cognitive functioning than the short-term storage component. As Matzel and Kolata (2010) stated: "This led to the conclusion that working memory capacity, but not short-term memory duration, was related to the animals' performance on the learning test battery" (p. 26).

One final example based on a variation on the classic Stroop paradigm (Stroop, 1935) provides evidence for the link between attention control and general intelligence in mice. Kolata et al. (2007) used a modified Stroop paradigm with two subtasks, which the mice received training in until they demonstrated errorless performance. In the visual discrimination subtask, the mice were placed in a visual discrimination box and trained to select a target stimulus from a set of three visual stimuli. In the odor discrimination subtask, the mice were placed in an odor discrimination box and trained to select a target stimulus from a set of three scents. On interference trials, the mice were placed either in the visual discrimination box with odor distractors, or in the odor discrimination box with visual distractors. Correct responses occurred when the mice selected the correct target stimulus for the discrimination box they had been placed in. Kolata et al. (2007) found that error rates increased substantially on interference trials and varied widely across individuals. More importantly, they found that performance under interference was strongly related to overall cognitive ability $(r=.50)$.

Using a similar Stroop paradigm, Crawford et al. (2020) found that older mice performed significantly worse under conditions of interference than younger mice. By contrast, both groups of mice performed equivalently on the individual subtasks, which did not require disengaging from previously learned associations. This pattern of results is particularly exciting, because it aligns with research on humans demonstrating that older adults exhibit deficits in cognitive 
control functions, including attention control (Braver \& Barch, 2002). Crawford et al.'s (2020) results suggest that cognitive aging in mice is related to deficits in disengaging from previously learned behavioral tendencies. Taken together, research using the modified Stroop paradigm has revealed that similar and homologous tasks can be used to measure attention control in mice and humans, with results that are broadly consistent across species.

\section{Conclusion}

To sum up, research on individual differences in intelligence in humans and mice provides converging evidence for a link between attention control and general intelligence. Attention control reflects the ability to regulate information processing in service of goals via information maintenance and disengagement - in other words, to maintain focus on task-relevant information and resist attentional capture from task-irrelevant thoughts and events. In research on humans, evidence suggests that attention control can largely explain the positive relationships between working memory capacity, fluid intelligence, and sensory discrimination ability. In research on mice, attention control has been closely linked to general cognitive ability as measured by a broad variety of tasks involving route learning and problem solving. More than one hundred years after Spearman's (1904) discovery of a general factor underpinning performance in cognitive domains, research has revealed at least one plausible explanation for the positive manifold observed among broad cognitive abilities. Nevertheless, efforts to find converging and disconfirmatory evidence across species stands to improve not only our understanding of attention control, but cognition in general. 


\section{References}

Aldinger, K. A., Sokoloff, G., Rosenberg, D. M., Palmer, A. A., \& Millen, K. J. (2009). Genetic variation and population substructure in outbred CD-1 mice: implications for genomewide association studies. PloS one, 4(3), e4729.

Ambrosini, E., Arbula, S., Rossato, C., Pacella, V., \& Vallesi, A. (2019). Neuro-cognitive architecture of executive functions: A latent variable analysis. Cortex, 119, 441-456.

Arden, R., \& Adams, M. J. (2016). A general intelligence factor in dogs. Intelligence, 55, 79-85.

Ashton, B. J., Ridley, A. R., Edwards, E. K., \& Thornton, A. (2018). Cognitive performance is linked to group size and affects fitness in Australian magpies. Nature, 554(7692), 364367.

Baddeley, A. (1992). Working memory. Science, 255(5044), 556-559.

Baddeley, A. (2000). The episodic buffer: a new component of working memory? Trends in cognitive sciences, 4, 417-423.

Baddeley, A. D., \& Hitch, G. J. (1974). Working memory. In G. H. Bower (Ed.), The psychology of learning and motivation Vol. 8 (pp. 47-89). New York: Academic Press.

Braver, T. S., \& Barch, D. M. (2002). A theory of cognitive control, aging cognition, and neuromodulation. Neuroscience \& Biobehavioral Reviews, 26, 809-817.

Burgoyne, A. P., \& Engle, R. W. (in press). Attention control: A cornerstone of higher-order cognition. Current Directions in Psychological Science.

Carroll, J. B. (1993). Human cognitive abilities: A survey of factor-analytic studies. Cambridge University Press.

Cole, E. F., Cram, D. L., \& Quinn, J. L. (2011). Individual variation in spontaneous problemsolving performance among wild great tits. Animal Behaviour, 81, 491-498. 
Conway, A. R., Cowan, N., \& Bunting, M. F. (2001). The cocktail party phenomenon revisited: The importance of working memory capacity. Psychonomic Bulletin \& Review, 8, 331335.

Conway, A. R., Cowan, N., Bunting, M. F., Therriault, D. J., \& Minkoff, S. R. (2002). A latent variable analysis of working memory capacity, short-term memory capacity, processing speed, and general fluid intelligence. Intelligence, 30, 163-183.

Kovacs, K., \& Conway, A. R. (2016). Process overlap theory: A unified account of the general factor of intelligence. Psychological Inquiry, 27, 151-177.

Crawford, D. W., Bendrath, S., Manzano, M. D., Mehta, A., Patel, H. M., Piela, M. C., Sauce, B., \& Matzel, L. D. (2020). Intelligence demands flexibility: Individual differences in attentional disengagement strongly predict the general cognitive ability of mice. Learning and Motivation, 71, 101657.

Cronbach, L. J. (1957). The two disciplines of scientific psychology. American Psychologist, 12, 671-684.

Dickens, W. T., \& Flynn, J. R. (2001). Heritability estimates versus large environmental effects: the IQ paradox resolved. Psychological Review, 108, 346-369.

Draheim, C., Tsukahara, J. S., Martin, J. D., Mashburn, C. A., \& Engle, R. W. (2020). A toolbox approach to improving the measurement of attention control. Journal of Experimental Psychology: General. https://doi.org/10.1037/xge0000783

Engle, R. W. (2002). Working memory capacity as executive attention. Current Directions in Psychological Science, 11, 19-23.

Engle, R. W. (2018). Working memory and executive attention: A revisit. Perspectives on Psychological Science, 13, 190-193. 
Engle, R. W., \& Kane, M. J. (2004). Executive attention, working memory capacity, and a twofactor theory of cognitive control. Psychology of Learning and Motivation, 44, 145-200.

Engle, R. W., Tuholski, S. W., Laughlin, J. E., \& Conway, A. R. (1999). Working memory, short-term memory, and general fluid intelligence: a latent-variable approach. Journal of Experimental Psychology: General, 128, 309-331.

Eriksen, B. A., \& Eriksen, C. W. (1974). Effects of noise letters upon the identification of a target letter in a nonsearch task. Perception \& Psychophysics, 16, 143-149.

Galsworthy, M. J., Paya-Cano, J. L., Monleon, S., \& Plomin, R. (2002). Evidence for general cognitive ability $(\mathrm{g})$ in heterogeneous stock mice and an analysis of potential confounds. Genes, Brain and Behavior, 1, 88-95.

Gottfredson, L. S. (1998). The general intelligence factor. Scientific American (pp. 24-30).

Hallett, P. E. (1978). Primary and secondary saccades to goals defined by instructions. Vision Research, 18, 1279-1296.

Heitz, R. P., \& Engle, R. W. (2007). Focusing the spotlight: Individual differences in visual attention control. Journal of Experimental Psychology: General, 136, 217-240.

Jensen, A. R. (1998). The g factor: The science of mental ability (Vol. 648). Westport, CT: Praeger.

Johnson-Ulrich, L., Holekamp, K. E., \& Hambrick, D. Z. (2020). Innovative problem-solving in wild hyenas is reliable across time and contexts. Scientific Reports, 10, 1-12.

Kane, M. J., Bleckley, M. K., Conway, A. R., \& Engle, R. W. (2001). A controlled-attention view of working-memory capacity. Journal of Experimental Psychology: General, 130, $169-183$. 
Kane, M. J., Conway, A. R. A., Hambrick, D. Z., \& Engle, R. W. (2007). Variation in working memory capacity as variation in executive attention and control. In A. R. A. Conway, C. Jarrold, M. J. Kane, A. Miyake, \& J. N. Towse (Eds.), Variation in working memory (pp. 21-48). New York, NY: Oxford University Press.

Kane, M. J., \& Engle, R. W. (2003). Working-memory capacity and the control of attention: the contributions of goal neglect, response competition, and task set to Stroop interference. Journal of Experimental Psychology: General, 132, 47-70.

Kane, M. J., Hambrick, D. Z., Tuholski, S. W., Wilhelm, O., Payne, T. W., \& Engle, R. W. (2004). The generality of working memory capacity: a latent-variable approach to verbal and visuospatial memory span and reasoning. Journal of Experimental Psychology: General, 133, 189-217.

Kline, R. B. (2015). Principles and practice of structural equation modeling. Guilford publications.

Kolata, S., Light, K., Grossman, H. C., Hale, G., \& Matzel, L. D. (2007). Selective attention is a primary determinant of the relationship between working memory and general learning ability in outbred mice. Learning \& Memory, 14, 22-28.

Kolata, S., Light, K., Townsend, D. A., Hale, G., Grossman, H. C., \& Matzel, L. D. (2005). Variations in working memory capacity predict individual differences in general learning abilities among genetically diverse mice. Neurobiology of Learning and Memory, 84, 241-246.

Kolata, S., Light, K., Wass, C. D., Colas-Zelin, D., Roy, D., \& Matzel, L. D. (2010). A dopaminergic gene cluster in the prefrontal cortex predicts performance indicative of general intelligence in genetically heterogeneous mice. PLoS One, 5(11), e14036. 
Matzel, L. D., Han, Y. R., Grossman, H., Karnik, M. S., Patel, D., Scott, N., Specht, S. M., \& Gandhi, C. C. (2003). Individual differences in the expression of a "general" learning ability in mice. Journal of Neuroscience, 23, 6423-6433.

Matzel, L. D., \& Kolata, S. (2010). Selective attention, working memory, and animal intelligence. Neuroscience \& Biobehavioral Reviews, 34, 23-30.

McGrew, K. S. (2009). CHC theory and the human cognitive abilities project: Standing on the shoulders of the giants of psychometric intelligence research. Intelligence, 37, 1-10.

Moray, N. (1959). Attention in dichotic listening: Affective cues and the influence of instructions. Quarterly Journal of Experimental Psychology, 11, 56-60.

Plomin, R. (2017). Foreword. In The Science of Expertise: Behavioral, Neural, and Genetic Approaches to Complex Skill. D.Z. Hambrick, G. Campitelli \& B.N. Macnamara (Eds.) (pp. xiv-xvii). New York: Routledge.

Raven, J. C., \& Court, J. H. (1998). Raven's progressive matrices and vocabulary scales. Oxford, UK: Oxford Psychologists Press.

Savi, A. O., Marsman, M., van der Maas, H. L., \& Maris, G. K. (2019). The wiring of intelligence. Perspectives on Psychological Science, 14, 1034-1061.

Shipstead, Z., Harrison, T. L., \& Engle, R. W. (2016). Working memory capacity and fluid intelligence: Maintenance and disengagement. Perspectives on Psychological Science, 11, 771-799.

Spearman, C. E. (1904). General Intelligence, objectively determined and measured. American Journal of Psychology, 15, 201-293.

Stroop, J. R. (1935). Studies of interference in serial verbal reactions. Journal of Experimental Psychology, 18, 643-662. 
Thorndike, R. L. (1935). Organization of behavior in the albino rat. Genetic Psychology Monographs, 17, 1-70.

Tsukahara, J. S., Harrison, T. L., Draheim, C., Martin, J. D., \& Engle, R. W. (2020). Attention control: The missing link between sensory discrimination and intelligence. Attention, Perception, \& Psychophysics. https://doi.org/10.3758/s13414-020-02044-9

Unsworth, N., Heitz, R. P., Schrock, J. C., \& Engle, R. W. (2005). An automated version of the operation span task. Behavior Research Methods, 37, 498-505.

Unsworth, N., Schrock, J. C., \& Engle, R. W. (2004). Working memory capacity and the antisaccade task: individual differences in voluntary saccade control. Journal of Experimental Psychology: Learning, Memory, and Cognition, 30, 1302-1321. 